\title{
Odd-Even Graceful Labeling in Duplicate Graphs of certain Path and Star Related Graphs
}

\author{
S. Christopher ${ }^{1}$, P. Vijaya kumar ${ }^{2}$ and K. Thulukkanam ${ }^{3}$ \\ ${ }^{1}$ Department of Mathematics, Voorhees College, Vellore - 632001, India \\ christyjoan2003@gmail.com \\ ${ }^{2}$ Department of Mathematics, Sriram Engineering College, Perumalpattu - 602024. \\ vijaysanthosh2002@gmail.com \\ ${ }^{3}$ Department of Mathematics, Dr. Ambedkar Govt. Arts College, Chennai 600039 \\ thulukkanamk@gmail.com
}

\begin{abstract}
In this paper, we prove that the extended duplicate graphs of star graph, bistar graph, double star graph, path graph, comb graph and twig graph admit odd-even graceful labeling labeling.
\end{abstract}

AMS Subject Classification: $05 \mathrm{C} 78$

Key Words: Graph labeling, duplicate graph, odd-even graceful labeling.

\section{Introduction}

The concept of graph labeling was introduced by Rosa in 1967. A graph labeling is an assignment of integers to the vertices or edges or both subject to certain condition(s). If the domain of the mapping is the set of vertices (or edges), then the labeling is called a vertex labeling (or an edge labeling). In the intervening years various labeling of graphs have been investigated in 
over 2000 papers [1]. The concept of duplicate graph was introduced by Sampath kumar and he proved many results on it 2]. E. Sampath kumar introduced the concept of duplicate graph and studied the charactarization of the the duplicate graphs [2]. Odd Even graceful was introduced by R Sridevi, S Navaneethakrishnan, A Nagarajan, K Nagarajan 3. Thirusangu, Ulaganathan and Selvam, have proved that the duplicate graph of a path graph $P_{m}$ is Cordial [6]. Thirusangu, Ulaganathan and Vijaya kumar have proved that the duplicate graph of Ladder graph $L_{m}, m \geq 2$, is cordial, total cordial and prime cordial[ $[5$. Vijaya kumar, Ulaganathan and Thirusangu, proved the existence of 3- Equitable and 3 - Cordial Labeling in Duplicate Graph of Some Graphs [6].

\section{MAIN RESULTS}

Definition 1. Let $G(V, E)$ be a graph with p vertices and q edges. A function $f: V \rightarrow$ $\{1,3,5, \ldots, 2 q+1\}$ is called an odd-even graceful labeling, when each edge $u v$ is assigned the label $|f(u)-f(v)|$ such that the resulting edge labels are $\{2,4,6, \ldots, 2 q\}$. A graph which admits an odd-even graceful labelingis called an odd-evengraceful graph. [3].

\subsection{Algorithm - OEGS}

$\mathrm{V} \leftarrow\left\{v_{1}, v_{2}, \ldots, v_{m}, v_{1}^{\prime}, v_{2}^{\prime}, \ldots, v_{m}^{\prime}\right\}$.

$\mathrm{E} \leftarrow\left\{e_{1}, e_{2}, \ldots, e_{m}, e_{1}^{\prime}, e_{2}^{\prime}, \ldots, e_{m-1}^{\prime}\right\}$.

For $1 \leq k \leq m-1$

$v_{k} \leftarrow 2 k-1, v_{k}^{\prime} \leftarrow 2 m-2 k-1 ;$

Theorem 2. The extended duplicate graph of the star graph $E D G\left(S_{m}\right), m \geq 2$ admits odd - even graceful labeling.

Proof. Let $\left\{v_{1}, v_{2}, \ldots, v_{m}, v_{1}^{\prime}, v_{2}^{\prime}, \ldots, v_{m}^{\prime}\right\}$ and $\left\{e_{1}, e_{2}, \ldots, e_{m}, e_{1}^{\prime}, e_{2}^{\prime}, \ldots, e_{m-1}^{\prime}\right\}$ be the set of vertices and the edges of the $E D G\left(S_{m}\right)$. Using the algorithm OEGS, the $2 m$ vertices are labeled using 1 , $3,5, \ldots, 4 m-1(=2 q+1)$. Using the induced function $f^{*}(u v)=|f(u)-f(v)|$, the $m-1$ edges namely $e_{m-1}^{\prime}, e_{m-2}^{\prime}, e_{m-3}^{\prime}, \ldots, e_{2}^{\prime}, e_{1}^{\prime}$ receive labels $2,4,6, \ldots, 2 m-4,2 m-2$ respectively, the edge $e_{2 m}$ receives label $2 m$ and the $m-1$ edges namely $e_{1}, e_{2}, e_{3}, \ldots, e_{m-2}, e_{m-1}$ receive labels $2 m+2$, $2 m+4,2 m+6, \ldots, 4 m-4,4 m-2(=2 q)$. Thus the $2 m-1$ edges are labeled with $2,4,6, \ldots$, 
$4 m-2(=2 q)$. Hence, the extended duplicate graph of the star graph $E D G\left(S_{m}\right), m \geq 2$ admits odd-even graceful labeling.

\section{$2.2 \quad$ Algorithm - OEGBS}

$\mathrm{V} \leftarrow\left\{v_{1}, v_{2}, \ldots, v_{2 m+2}, v_{1}^{\prime}, v_{2}^{\prime}, \ldots, v_{2 m+2}^{\prime}\right\}$
$\mathrm{E} \leftarrow\left\{e_{1}, e_{2}, \ldots, e_{2 m+2}, e_{1}^{\prime}, e_{2}^{\prime}, \ldots, e_{2 m+1}^{\prime}\right\}$

Fix $v_{1} \leftarrow 1, v_{1}^{\prime} \leftarrow 4 m+5$;

For $1 \leq k \leq m+1$

$v_{k+1}^{\prime} \leftarrow 8 m-2 k+9$;

For $1 \leq k \leq m$

$v_{m+k+2}^{\prime} \leftarrow 6 m-2 k+7$;

For $1 \leq k \leq 2 m+1$

$v_{k+1} \leftarrow 4 m-2 k+5$;

Theorem 3. The extended duplicate graph of the bistar graph $E D G\left(B S_{m}\right), m \geq 2$ admits odd - even graceful labeling.

Proof. Let $\left\{v_{1}, v_{2}, \ldots, v_{2 m+2}, v_{1}^{\prime}, v_{2}^{\prime}, \ldots, v_{2 m+2}^{\prime}\right\}$ and $\left\{e_{1}, e_{2}, \ldots, e_{2 m+2}, e_{1}^{\prime}, e_{2}^{\prime}, \ldots, e_{2 m+1}^{\prime}\right\}$ be the set of vertices and the edges of $E D G\left(C B_{m}\right)$. Using the algorithm OEGBS, $4 m+4$ vertices are labeled using $1,3,5, \ldots, 8 m+7(=2 q+1)$. Using the induced function $f^{*}$ defined in theorem 2 , the $m+1$ edges namely $e_{1}, e_{2}, \ldots, e_{m}, e_{m+1}$ receive labels $8 m+6(=2 q), 8 m+4,8 m+2, \ldots, 6 m+8,6 m+6$ respectively, the $m$ edges namely $e_{2 m+1}^{\prime}, e_{2 m}^{\prime}, e_{2 m-1}^{\prime}, \ldots, e_{m+3}^{\prime}, e_{m+2}^{\prime}$ receive labels $6 m+4,6 m+2$, $6 m, \ldots, 4 m+8,4 m+6$ respectively, the $m$ edges namely $e_{m+2}, e_{m+3}, e_{m+4}, \ldots, e_{2 m}, e_{2 m+1}$ receive labels $4 m+2,4 m, 4 m-2, \ldots, 2 m+6,2 m+4$ respectively, the $m$ edges namely $e_{m+1}^{\prime}, e_{m}^{\prime}, e_{m-1}^{\prime}, \ldots$, $e_{2}^{\prime}, e_{1}^{\prime}$ receive labels $2 m+2,2 m, 2 m-2, \ldots, 4,2$ respectively and the edge $e_{2 m+2}$ receives label $4 m+4$. Thus the $4 m+3$ edges are labeled with $2,4,6, \ldots, 8 m+6(=2 q)$. Hence the extended duplicate graph of the bistar graph $E D G\left(B S_{m}\right), m \geq 2$ admits odd - even graceful labeling.

\section{$2.3 \quad$ Algorithm - OEGDS}

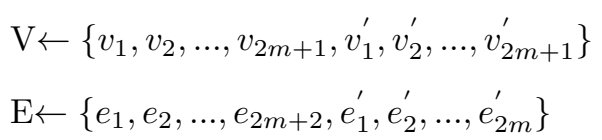

Fix $v_{1} \leftarrow 1$. For $1 \leq k \leq 2 m$ 
$v_{k+1} \leftarrow 6 m-2 k+3$

For $1 \leq k \leq m+1$

$v_{m-k+2}^{\prime} \leftarrow 8 m-2 k+5$

For $1 \leq k \leq m+1$

$v_{m+k+1}^{\prime} \leftarrow 2 k+1$

Theorem 4. The extended duplicate graph of the double star graph EDG(DSm), $\geq 2$ admits odd - even graceful labeling.

Proof. Let $\left\{v_{1}, v_{2}, \ldots, v_{2 m+1}, v_{1}^{\prime}, v_{2}^{\prime}, \ldots, v_{2 m+1}^{\prime}\right\}$ and $\left\{e_{1}, e_{2}, \ldots, e_{2 m+1}, e_{1}^{\prime}, e_{2}^{\prime}, \ldots, e_{2 m}^{\prime}\right\}$ be the set of vertices and the edges of $E D G\left(D S_{m}\right)$. Using the algorithm OEGDS, the $4 m+2$ vertices are labeled using $1,3,5, \ldots, 8 m+3(=2 q+1)$. Using the induced function $f^{*}$ defined in theorem 2 , the $m$ edges namely $e_{1}^{\prime}, e_{2}^{\prime}, e_{3}^{\prime}, \ldots, e_{m-1}^{\prime}, e_{m}^{\prime}$ receive labels $2,4,6, \ldots, 2 m-2,2 m$ respectively, the $m$ edges namely $e_{2 m}, e_{2 m-1}, e_{2 m-2}, \ldots, e_{m+2}, e_{m+1}$ receive labels $2 m+2,2 m+6,2 m+10, \ldots$, $6 m-6,6 m-2$ respectively, the $m$ edges namely $e_{m+1}^{\prime}, e_{m+2}^{\prime}, e_{m+3}^{\prime}, \ldots, e_{2 m-1}^{\prime}, e_{2 m}^{\prime}$ receive labels $2 m+4,2 m+8,2 m+12, \ldots, 6 m-4,6 m$ respectively, the $m$ edges namely $e_{1}, e_{2}, e_{3}, \ldots, e_{m-1}$, $e_{m}$ receive labels $6 m+4,6 m+6,6 m+8, \ldots, 8 m, 8 m+2$ respectively and the edge $e_{2 m+1}$ receives the label $6 m+2$. Thus the $4 m+1(=2 q)$ edges are labeled with $2,4,6, \ldots, 8 m+2(=2 q)$. Hence the extended duplicate graph of the double star graph $E D G\left(D S_{m}\right), m \geq 2$ admits odd - even graceful labeling.

Illustration:
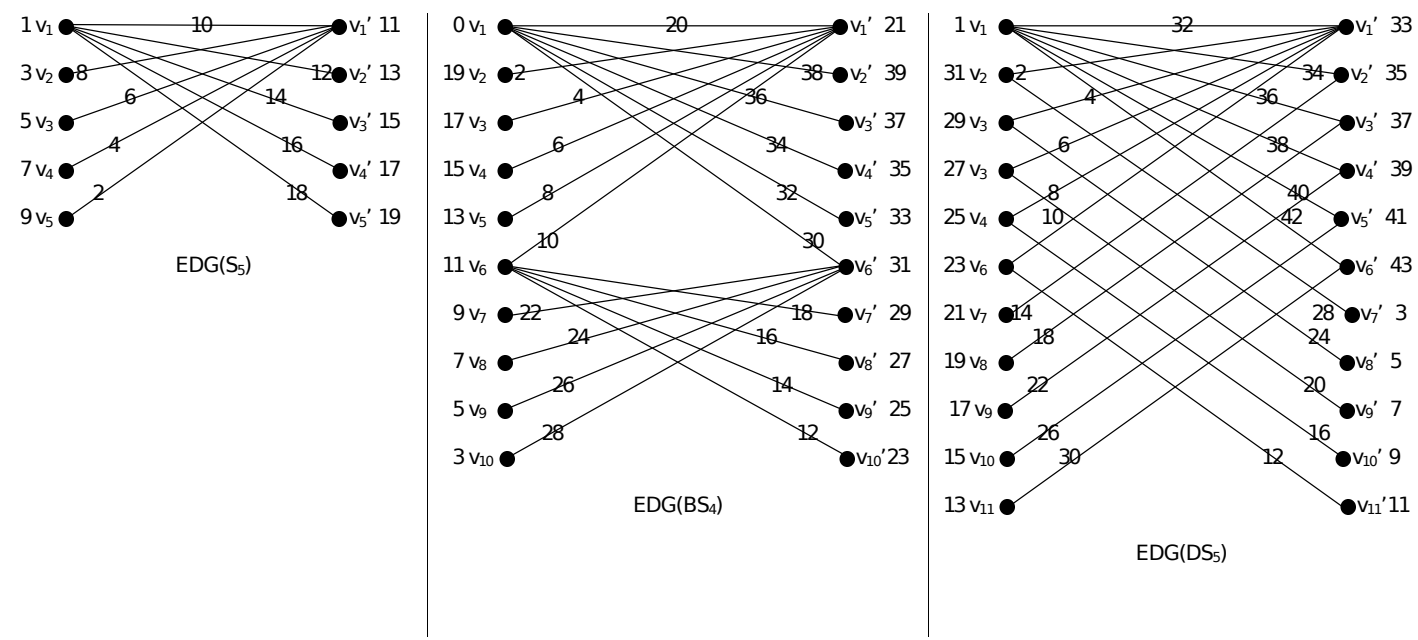

captionFigure 1. Odd-Even Graceful labeling in $E D G\left(S_{5}\right), E D G\left(B S_{4}\right)$ andEDG $\left(D S_{5}\right)$ 


\subsection{Algorithm - OEGP}

$$
\begin{aligned}
& \mathrm{V} \leftarrow\left\{v_{1}, v_{2}, \ldots, v_{m+1}, v_{1}^{\prime}, v_{2}^{\prime}, \ldots, v_{m+1}^{\prime}\right\} \\
& \mathrm{E} \leftarrow\left\{e_{1}, e_{2}, \ldots, e_{m+1}, e_{1}^{\prime}, e_{2}^{\prime}, \ldots, e_{m}^{\prime}\right\}
\end{aligned}
$$

Case (i): when $m$ is odd

For $1 \leq k \leq \frac{m+1}{2}$

$v_{2 k} \leftarrow 2 k-1, v_{2 k-1} \leftarrow 2 m-2 k+3$

$v_{2 k}^{\prime} \leftarrow 2 m+2 k+1, v_{2 k-1}^{\prime} \leftarrow 4 m-2 k+5$

Case (ii): when $m$ is even

For $1 \leq k \leq \frac{m}{2}$

$v_{2 k} \leftarrow 2 k-1, v_{2 k-1} \leftarrow 2 m-2 k+3$

$v_{2 k}^{\prime} \leftarrow 2 m+2 k+1, v_{2 k+1}^{\prime} \leftarrow 4 m-2 k+5$;

Theorem 5. The extended duplicate graph of the path graph EDG $\left(P_{m}\right), m \geq 2$ admits odd - even graceful labeling.

Proof. Let $\left\{v_{1}, v_{2}, \ldots, v_{m+1}, v_{1}^{\prime}, v_{2}^{\prime}, \ldots, v_{m+1}^{\prime}\right\}$ and $\left\{e_{1}, e_{2}, \ldots, e_{m+1}, e_{1}^{\prime}, e_{2}^{\prime}, \ldots, e_{m}^{\prime}\right\}$ be the set of vertices and the edges of the $E D G\left(P_{m}\right)$.

Case (i): when $m$ is odd

Using the algorithm OEGP, the $2 m+2$ vertices are labeled using $1,3,5, \ldots, 4 m+3(=2 q+1)$.

Using the induced function $f^{*}$ defined in theorem 2 , the $m$ edges namely, $e_{1} e_{2}^{\prime}, e_{3}, e_{4}^{\prime}, e_{5}, e_{6}^{\prime}, \ldots$, $e_{m-2}, e_{m-1}^{\prime}, e_{m}$ receive labels $2,4,6,8,10,12, \ldots, 2 m-4,2 m-2,2 m$ respectively, the $m$ edges namely $e_{1}^{\prime}, e_{2}, e_{3}^{\prime}, e_{4}, e_{5}^{\prime}, e_{6}, \ldots, e_{m-2}^{\prime}, e_{m-1}, e_{m}^{\prime}$ receive labels $4 m+2(=2 q), 4 m, 4 m-2,4 m-4$, $4 m-6,4 m-8, \ldots, 2 m+8,2 m+6,2 m+4$ respectively and the edge $e_{m+1}$ receives the label $2 m+2$. Thus the $2 m+1(=q)$ edges are labeled with $2,4,6, \ldots, 4 m+2(=2 q)$.

Case (ii): when $m$ is even

Using the algorithm OEGP, the $2 m+2$ vertices are labeled using $1,3,5, \ldots, 4 m+3(=2 q+1)$. Using the induced function $f^{*}$ defined in theorem 2 , the $m$ edges namely, $e_{1} e_{2}^{\prime}, e_{3}, e_{4}^{\prime}, e_{5}, e_{6}^{\prime}, \ldots$, $e_{m-3}, e_{m-2}^{\prime}, e_{m-1}, e_{m}^{\prime}$ receive labels $2,4,6,8,10,12, \ldots, 2 m-6,2 m-4,2 m-2,2 m$ respectively, the $m$ edges namely $e_{1}^{\prime}, e_{2}, e_{3}^{\prime}, e_{4}, e_{5}^{\prime}, e_{6}, \ldots, e_{m-3}^{\prime}, e_{m-2}, e_{m-1}^{\prime}, e_{m}$ receive labels $4 m+2(=2 q), 4 m$, $4 m-2,4 m-4,4 m-6,4 m-8, \ldots, 2 m+10,2 m+8,2 m+6,2 m+4$ respectively and the edge $e_{m+1}$ receives the label $2 m+2$. Thus the $2 m+1(=q)$ edges are labeled with $2,4,6, \ldots, 4 m+2(=2 q)$. Hence the extended duplicate graph of the path graph $E D G\left(P_{m}\right), m \geq 2$ admits odd - even 
graceful labeling.

\subsection{Algorithm - OEGCB}

$\mathrm{V} \leftarrow\left\{v_{1}, v_{2}, \ldots, v_{2 m}, v_{1}^{\prime}, v_{2}^{\prime}, \ldots, v_{2 m}^{\prime}\right\}$

$\mathrm{E} \leftarrow\left\{e_{1}, e_{2}, \ldots, e_{2 m}, e_{1}^{\prime}, e_{2}^{\prime}, \ldots, e_{2 m-1}^{\prime}\right\}$

Case (i): when $m$ is odd

Fix $v_{1} \leftarrow 1, v_{1}^{\prime} \leftarrow 4 m+1$

For $1 \leq k \leq \frac{m-1}{2}$

$v_{4 k} \leftarrow 4 k-1, v_{4 k+1} \leftarrow 4 k+1, v_{4 k-2} \leftarrow 4 m-4 k+3, v_{4 k-1} \leftarrow 4 m-4 k+1$,

$v_{4 k}^{\prime} \leftarrow 4 m-4 k+7, v_{4 k+1}^{\prime} \leftarrow 4 m-4 k+9, v_{4 k-2}^{\prime} \leftarrow 8 m-4 k+3, v_{4 k-1}^{\prime} \leftarrow 8 m-4 k+1, ;$

Case (ii): when $m$ is even

Fix $v_{1} \leftarrow 1, v_{1}^{\prime} \leftarrow 4 m+1$

For $1 \leq k \leq \frac{m-2}{2}$

$v_{4 k} \leftarrow 4 k-1, v_{4 k+1} \leftarrow 4 k+1$

$v_{4 k}^{\prime} \leftarrow 4 m+4 k-1, v_{4 k+1}^{\prime} \leftarrow 4 m+4 k+1$

For $1 \leq k \leq \frac{m}{2}$

$v_{4 k-2} \leftarrow 4 m-4 k+3, v_{4 k-1} \leftarrow 4 m-4 k+1$

$v_{4 k-2}^{\prime} \leftarrow 8 m-4 k+3, v_{4 k-1}^{\prime} \leftarrow 8 m-4 k+1$

For $k=2 m v_{k} \leftarrow 2 m, v_{k}^{\prime} \leftarrow 6 m+1$;

Theorem 6. The extended duplicate graph of the comb graph $E D G\left(C B_{m}\right), m \geq 2$ admits odd - even graceful labeling.

Proof. Let $\left\{v_{1}, v_{2}, \ldots, v_{2 m}, v_{1}^{\prime}, v_{2}^{\prime}, \ldots, v_{2 m}^{\prime}\right\}$ and $\left\{e_{1}, e_{2}, \ldots, e_{2 m}, e_{1}^{\prime}, e_{2}^{\prime}, \ldots, e_{2 m-1}^{\prime}\right\}$ be the set of vertices and the edges of the $E D G\left(C B_{m}\right)$.

Case (i): when $m$ is odd

Using the algorithm OEGCB, the $4 m$ vertices are labeled using $1,3,5, \ldots, 8 m-1(=2 q+1)$. Using the induced function $f^{*}$ defined in theorem 2 , the $2 m-2$ edges namely $e_{1}^{\prime}, e_{2}^{\prime}, e_{3}, e_{4}, e_{5}^{\prime}, e_{6}^{\prime}, e_{7}$, $e_{8}, \ldots, e_{2 m-5}^{\prime}, e_{2 m-4}^{\prime}, e_{2 m-3}, e_{2 m-2}$ receive labels $2,4,6,8,10,12,14,16, \ldots, 4 m-10,4 m-8$, $4 m-6,4 m-4$ respectively, the 3 edges $e_{2 m-1}^{\prime}, e_{2 m}, e_{2 m-1}$ receive labels $4 m-2,4 m, 4 m+2$ respectively and the $2 m-2$ edges namely $e_{2 m-2}^{\prime}, e_{2 m-3}^{\prime}, e_{2 m-4}, e_{2 m-5}, \ldots, e_{4}^{\prime}, e_{3}^{\prime}, e_{2}, e_{1}$ receive labels $4 m+4,4 m+6,4 m+8,4 m+10, \ldots, 8 m-8,8 m-6,8 m-4,8 m-2$ respectively. Thus the 
$4 m-1(=q)$ edges are labeled with $2,4,6,8, \ldots, 8 m-2(=2 q)$.

Case (ii): when $m$ is even

Using the algorithm OEGCB, the $4 m$ vertices are labeled using $1,3,5, \ldots, 8 m-1(=2 q+1)$. Using the induced function $f^{*}$ defined in theorem 2 , the $2 m-2$ edges namely $e_{1}^{\prime}, e_{2}^{\prime}, e_{3}, e_{4}, e_{5}^{\prime}, e_{6}^{\prime}, e_{7}$, $e_{8}, \ldots, e_{2 m-7}^{\prime}, e_{2 m-6}^{\prime}, e_{2 m-5}, e_{2 m-4}$ receive labels $2,4,6,8,10,12,14,16, \ldots, 4 m-14,4 m-12$, $4 m-10,4 m-8,4 m-6,4 m-4$ respectively, the 2 edges $e_{2 m-1}, e_{2 m}$ receive labels $4 m-2,4 m$ respectively and the edge $e_{2 m-1}^{\prime}$ receives label $4 m+2$ and the $2 m-2$ edges namely $e_{2 m-2}, e_{2 m-3}$, $e_{2 m-4}^{\prime}, e_{2 m-5}^{\prime}, e_{2 m-6}, e_{2 m-7}, \ldots, e_{4}^{\prime}, e_{3}^{\prime}, e_{2}, e_{1}$ receive labels $4 m+4,4 m+6,4 m+8,4 m+10, \ldots$, $8 m-8,8 m-6,8 m-4,8 m-2$ respectively. Thus the $4 m-1(=q)$ edges are labeled with 2,4 , $6,8, \ldots, 8 m-2(=2 q)$.

Hence, the extended duplicate graph of the comb graph $E D G\left(C B_{m}\right), m \geq 2$ admits odd - even graceful labeling.

\subsection{Algorithm - OEGT}

$\mathrm{V} \leftarrow\left\{v_{1}, v_{2}, \ldots, v_{3 m+2}, v_{1}^{\prime}, v_{2}^{\prime}, \ldots, v_{3 m+2}^{\prime}\right\}$

$\mathrm{E} \leftarrow\left\{e_{1}, e_{2}, \ldots, e_{3 m+2}, e_{1}^{\prime}, e_{2}^{\prime}, \ldots, e_{3 m+1}^{\prime}\right\}$

Case (i): when $m$ is odd

Fix $v_{1} \leftarrow 6 m+3, v_{2} \leftarrow 1, v_{1}^{\prime} \leftarrow 12 m+7, v_{2}^{\prime} \leftarrow 6 m+5$

For $1 \leq k \leq \frac{m+1}{2}$

$v_{6 k-3} \leftarrow 6 m-6 k+7, v_{6 k-2} \leftarrow 6 m-6 k+5, v_{6 k-1} \leftarrow 6 m-6 k+3 ;$

$v_{6 k-3}^{\prime} \leftarrow 12 m-6 k+11, v_{6 k-2}^{\prime} \leftarrow 12 m-6 k+9, v_{6 k-1}^{\prime} \leftarrow 12 m-6 k+7$;

For $1 \leq k \leq \frac{m-1}{2}$

$v_{6 k} \leftarrow 6 k-3, v_{6 k+1} \leftarrow 6 k-1, v_{6 k+2} \leftarrow 6 k+1$

$v_{6 k}^{\prime} \leftarrow 6 m+6 k+1, v_{6 k+1}^{\prime} \leftarrow 6 m+6 k+3, v_{6 k+2}^{\prime} \leftarrow 6 m+6 k+7 ;$

Case (ii): when $m$ is even

Fix $v_{1} \leftarrow 6 m+3, v_{2} \leftarrow 1, v_{1}^{\prime} \leftarrow 12 m+7, v_{2}^{\prime} \leftarrow 6 m+5$

For $1 \leq k \leq \frac{m}{2}$

$v_{6 k-3} \leftarrow 6 m-6 k+7, v_{6 k-2} \leftarrow 6 m-6 k+5, v_{6 k-1} \leftarrow 6 m-6 k+3, v_{6 k} \leftarrow 6 k-3, v_{6 k+1} \leftarrow 6 k-1$,

$v_{6 k+2} \leftarrow 6 k+1 ;$

$v_{6 k-3}^{\prime} \leftarrow 12 m-6 k+11, v_{6 k-2}^{\prime} \leftarrow 12 m-6 k+9, v_{6 k-1}^{\prime} \leftarrow 12 m-6 k+7, v_{6 k}^{\prime} \leftarrow 6 m+6 k+1$,

$v_{6 k+1}^{\prime} \leftarrow 6 m+6 k+3, v_{6 k+2}^{\prime} \leftarrow 6 m+6 k+5$; 
Theorem 7. The extended duplicate graph of the twig graph $E D G\left(T_{m}\right), m \geq 2$ admits odd - even graceful labeling.

Proof. Let $\left\{v_{1}, v_{2}, \ldots, v_{3 m+2}, v_{1}^{\prime}, v_{2}^{\prime}, \ldots, v_{3 m+2}^{\prime}\right\}$ and $\left\{e_{1}, e_{2}, \ldots, e_{3 m+2}, e_{1}^{\prime}, e_{2}^{\prime}, \ldots, e_{3 m+1}^{\prime}\right\}$ be the set of vertices and the edges of the $E D G\left(T_{m}\right)$.

Case (i): when $m$ is odd

Using the algorithm OEGT, the $6 m+4$ vertices are labeled using $1,3,5, \ldots, 12 m+7(=2 q+1)$. Using the induced function $f^{*}$ defined in theorem 2 , the $3 m-3$ edges namely $e_{2}, e_{3}, e_{4}, e_{5}^{\prime}$, $e_{6}^{\prime}, e_{7}^{\prime}, e_{8}, e_{9}, e_{10}, e_{11}^{\prime}, e_{12}^{\prime}, e_{13}^{\prime}, \ldots, e_{3 m-7}^{\prime}, e_{3 m-6}^{\prime}, e_{3 m-5}^{\prime}, e_{3 m-4}, e_{3 m-3}, e_{3 m-2}$ receive labels $12 m+4$, $12 m+2,12 m, 12 m-2,12 m-4,12 m-6,12 m-8,12 m-10,12 m-12,12 m-14,12 m-16, \ldots$, $6 m+22,6 m+20,6 m+18,6 m+16,6 m+14,6 m+12$ respectively, the $3 m-3$ edges namely $e_{2}^{\prime}, e_{3}^{\prime}, e_{4}^{\prime}, e_{5}, e_{6}, e_{7}, e_{8}^{\prime}, e_{9}^{\prime}, e_{10}^{\prime}, e_{11}, e_{12}, e_{13}, \ldots, e_{3 m-7}, e_{3 m-6}, e_{3 m-5}, e_{3 m-4}^{\prime}, e_{3 m-3}^{\prime}, e_{3 m-2}^{\prime}$ receive labels $2,4,6,8,10,12,14,16,18,20,22,24, \ldots, 6 m-14,6 m-12,6 m-10,6 m-8,6 m-6,6 m-4$ respectively, the 6 edges namely $e_{3 m-1}^{\prime}, e_{3 m}^{\prime}, e_{3 m+1}^{\prime}, e_{3 m-1}, e_{3 m}, e_{3 m+1}$ receive labels $6 m-2,6 m$, $6 m+2,6 m+10,6 m+8,6 m+6$ respectively and the 3 edges namely $e_{1}, e_{1}^{\prime}, e_{3 m+2}$ receive labels $2,12 m+6(=2 q), 6 m+4$ respectively. Thus the $6 m+3(=q)$ edges are labeled with $2,4,6,8, \ldots$, $12 m+6(=2 q)$.

Case(ii): when $m$ is even

Using the algorithm OEGT, the $6 m+4$ vertices are labeled using $1,3,5, \ldots, 12 m+7(=2 q+1)$. Using the induced function $f^{*}$ defined in theorem 2 , the $3 m$ edges namely $e_{2}, e_{3}, e_{4}, e_{5}^{\prime}, e_{6}^{\prime}, e_{7}^{\prime}, e_{8}$, $e_{9}, e_{10}, e_{11}^{\prime}, e_{12}^{\prime}, e_{13}^{\prime}, \ldots, e_{3 m-4}, e_{3 m-3}, e_{3 m-2}, e_{3 m-1}^{\prime}, e_{3 m}^{\prime}, e_{3 m+1}^{\prime}$ receive labels $12 m+4,12 m+2$, $12 m, 12 m-2,12 m-4,12 m-6,12 m-8,12 m-10,12 m-12,12 m-14,12 m-16, \ldots, 6 m+16$, $6 m+14,6 m+12,6 m+10,6 m+8,6 m+6$ respectively, the $3 m$ edges namely $e_{2}^{\prime}, e_{3}^{\prime}, e_{4}^{\prime}, e_{5}, e_{6}, e_{7}$, $e_{8}^{\prime}, e_{9}^{\prime}, e_{10}^{\prime}, e_{11}, e_{12}, e_{13}, \ldots, e_{3 m-4}^{\prime}, e_{3 m-3}^{\prime}, e_{3 m-2}^{\prime}, e_{3 m-1}, e_{3 m}, e_{3 m+1}$, receive labels $2,4,6,8,10,12$, $14,16,18,20,22,24, \ldots, 6 m-2,6 m, 6 m+2$ and the 3 edges namely $e_{1}, e_{1}^{\prime}, e_{3 m+2}$ receive labels $2,12 m+6(=2 q), 6 m+4$ respectively. Thus the $6 m+3(=q)$ edges are labeled with $2,4,6,8, \ldots$, $12 m+6(=2 q)$.

Hence, the extended duplicate graph of the twig graph $E D G\left(T_{m}\right), m \geq 2$ admits odd - even graceful labeling.

Illustration: 


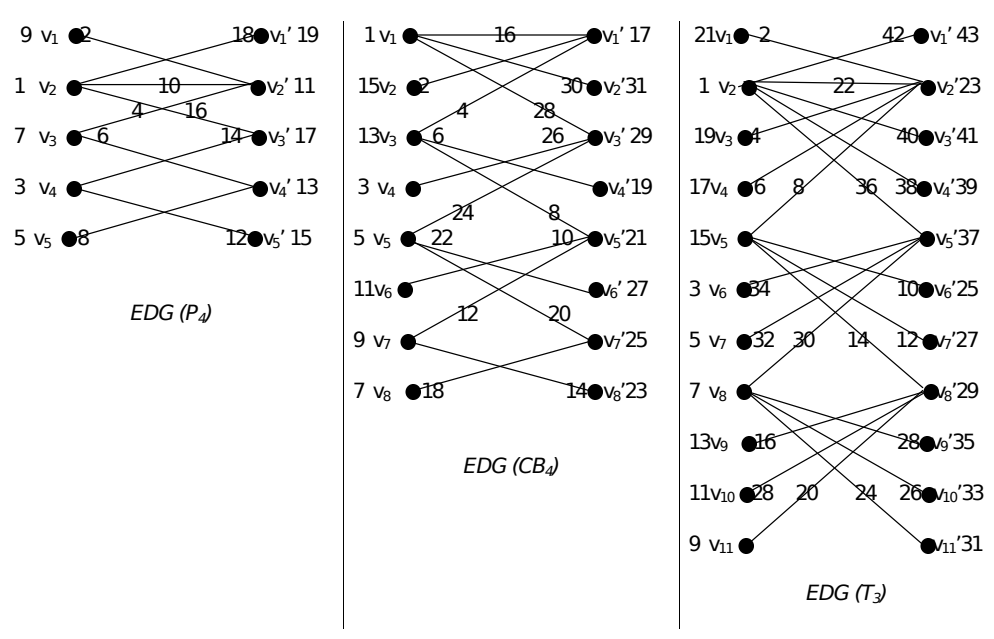

Figure 2. Odd-Even Graceful labeling in $E D G\left(P_{5}\right), E D G\left(C B_{4}\right)$ and $E D G\left(T_{3}\right)$

\section{Conclusion}

We have proved that extended duplicate graphs of star graph, bistar graph, double star graph, path graph, comb graph and twig graph admit odd - even graceful labeling.

\section{References}

[1] J.A. Gallian, A Dynamic Survey of Graph Labeling, The Electronic Journal of Combinatorics, DS6 (2016).

[2] E. Sampath kumar, On duplicate graphs, Journal of the Indian Math. Soc., 37 (1973), 285293.

[3] R. Sridevi, S. Navaneethakrishnan and K. Nagarajan, Odd-Even graceful graphs, J.Appl.Math. Informatics Vol.30 (2012), No. 5-6, pp. 913-923.

[4] K. Thirusangu, P.P. Ulaganathan and P. Vijaya kumar, Some cordial labeling of duplicate graph of ladder graph, Annals of Pure and Applied Mathematics, 8(2) (2014), 43-50.

[5] K. Thirusangu, P.P. Ulaganathan and B. Selvam. B, Cordial labeling in duplicate graphs, Int. J. Compute Math. Sci. Appl. Vol 4, Nos(1-2) (2010) 179186.

[6] Vijaya kumar. P, Ulaganathan. P.P and Thirusangu. K, 3Equitable and 3Cordial Labeling in Duplicate Graph of Some Graphs, International Journal of Mathematical Archive-6(12), 2015, 75-89. 